\title{
Longitudinal associations of age and prenatal lead exposure on cortisol secretion of 12-24 month-old infants from Mexico City
}

\author{
Marcela Tamayo y Ortiz ${ }^{1,2^{*}}$, Martha María Téllez-Rojo ${ }^{2}$, Rosalind J. Wright ${ }^{3,4,6}$, Brent A. Coull ${ }^{5}$ \\ and Robert O. Wrightt, 3,6
}

\begin{abstract}
Background: Cortisol has functions on homeostasis, growth, neurodevelopment, immune function and the stress response. Secretion follows a diurnal rhythm that mediates these processes. Our objective was to examine the association between prenatal lead exposure and infant diurnal cortisol rhythms.

Methods: We measured infant cortisol rhythms in saliva collected repeatedly over 2 days at either $12(n=255)$ or 18-24 ( $n=150)$ months of age. Prenatal lead exposure was assessed by measuring maternal pregnancy blood lead levels and early postnatal maternal bone lead content. We analyzed age-specific basal secretion and the association between trimester-specific and cumulative lead exposure with a) change in total diurnal cortisol and b) the shape of the cortisol curve across the length of the day.

Results: Our results showed age related differences in salivary cortisol secretion and an age dependent association with maternal lead exposure. In age-stratified models we saw an inverse association between lead and cortisol levels in 12-month-old infants and a positive association for 18-24-month-old infants. For the 12-month-old infants 2nd-trimester-lead $\geq 10 \mu \mathrm{g} / \mathrm{dL}$ was associated with $40 \%$ lower cortisol levels $(95 \% \mathrm{Cl}(-57,-16))$ and a significant change in the shape of the cortisol curve $(p=0.01)$, compared to infants with low blood lead levels $(<5 \mu \mathrm{g} / \mathrm{dL})$.
\end{abstract}

Conclusions: Basal cortisol secretion changes with age. Increased early gestation lead exposure alters diurnal cortisol rhythms and the association is modified by infant age, perhaps representing an early maturation of cortisol homeostasis.

Keywords: Lead, Cortisol, Epidemiology, Prenatal

\section{Background}

Endocrine disruption refers to environmental factors that interfere with hormone metabolism and homeostasis, processes that might explain developmental programming by prenatal chemical exposure $[1,2]$ ultimately impacting adult health and disease [3]. Cortisol is a critical metabolic hormone that mediates several homeostatic processes that

\footnotetext{
* Correspondence: marcela.tamayo@insp.mx

${ }^{1}$ National Council of Science and Technology, Center for Nutrition Research and Health, National Institute of Public Health, 7a. Cerrada de Fray Pedro de Gante \# 50, Col. Sección XVI Tlalpan, C.P. 14080 México D.F., Mexico

${ }^{2}$ Center for Research in Nutrition and Health, National Institute of Public Health, Universidad No. 655, Col. Santa María Ahuacatitlán, Cerrada los Pinos y Caminera, CP. 62100 Cuernavaca, Morelos, Mexico

Full list of author information is available at the end of the article
}

are essential to development and plays a direct role in neurodevelopment, growth/obesity and immune function. The hypothalamic-pituitary-adrenal (HPA) axis is the primary regulator of cortisol metabolism and its basal secretion; it mediates both stable day-to-day physiologic secretion of cortisol, and the increase in secretion that arises from environmental stimuli (danger, infection, diet, etc.) [4]. Understanding how the environment might program alterations in cortisol physiology requires measuring cortisol noninvasively (to avoid the stress of blood draws that can confound results), and repeatedly (to capture the diurnal variation in cortisol levels that naturally occurs).

Prenatal lead $(\mathrm{Pb})$ exposure has been consistently associated with a number of adverse health effects that 
overlap with cortisol function, including growth [5, 6] and altered neurodevelopment [7-9]. Research on environmental chemical toxicants and their effects on infant or early childhood HPA axis functioning which regulates cortisol excretion [10-12] remains limited. Studies on lead exposure and cortisol have mixed results, showing an increase or no change in cortisol production in 9 year old children and in occupationally exposed to lead male workers respectively [13-15]. Nevertheless, studies examining associations with exposure to other environmental chemicals in early development have suggested an increase in cortisol production and also suggest that effects may vary based on developmental stage of exposure and effect measurement of HPA function [16]. The present study expands upon this prior research by examining the relationship between prenatal exposure to $\mathrm{Pb}$ and basal HPA axis functioning (rather than the response to a stressor), as characterized by infant diurnal cortisol rhythms measured using timed salivary samples, in 12-24 month-old infants in a Mexican birth cohort. We are unaware of any data that establish norms for basal cortisol rhythms for these ages, and there is relatively little data on the timing of HPA axis maturation (although there is evidence of the establishment of the basic cortisol pattern (higher levels in the morning and lower levels late in the day) by 1 year of age, the current hypothesis is that it occurs around the 3rd year of life in humans [17]. Therefore, our main objectives were to investigate: a) if there is an age-dependent difference in the association of lead with cortisol rhythms, $b$ ) the association between lead and infant diurnal cortisol rhythms, and we hypothesized that higher prenatal lead exposure would be associated with increased cortisol production over the course of the day and $c$ ) if a specific window of prenatal susceptibility to lead could be identified. For objectives $a$ and $c$, we hypothesized that there would be a difference in the association depending on infant age and that a specific prenatal window of exposure would be detected, however we preferred not to define a direction or magnitude of the effect (objective a) or to point at a specific window (objective c) due to lack of human studies on this specific theme.

\section{Methods}

\section{Study population}

This study was conducted in the Programming Research in Obesity, Growth, Environment and Social Stressors (PROGRESS) birth cohort in Mexico City, previously described in more detail [18]. Briefly, women were invited to participate during their prenatal care visits at 4 clinics belonging to the Mexican Social Security System (Instituto Mexicano del Seguro Social [IMSS]) and were consented in a face-to-face interview and enrolled in their 2nd trimester of pregnancy. Between 2007 and 2011, 948 live infants were born and $760(80 \%)$ mother-infant pairs returned for follow-up visits between 6 and 24 months of age. The saliva collection kit was provided to all participants in follow-up (between 12 and 24 months) and the protocol was completed by 411 children of which 405 were included in the analyses. Participant characteristics did not differ significantly to those of non-participants $(p>0.05$ for all comparison tests of covariates between non-participants and participants) as shown in Table 1. Study protocols were approved by the institutional review boards of the Icahn School of Medicine at Mount Sinai, Harvard T. H. Chan School of Public Health, the National Institute of Public Health Mexico, the Mexican Social Security System, and the National Institute of Perinatology, Mexico. At each visit the study protocol was explained to women, who provided informed consent before any procedure was carried out.

\section{Maternal lead in blood}

Venous blood from women was drawn into royal blue trace metal vacutainer (Becton-Dickinson and Company, Franklin Lakes, New Jersey) tubes containing EDTA. Samples were collected from women in the second trimester $(2 \mathrm{~T})$ between 16 and 20 weeks of gestation and the third trimester $(3 \mathrm{~T})$ between 30 and 34 weeks. Samples were kept at $4{ }^{\circ} \mathrm{C}$ until analyzed. $\mathrm{BPb}$ concentrations were measured using a dynamic reaction cell inductively-coupled plasma mass spectrometer (Elan 6100; PerkinElmer, Norwalk, CT). Five replicate measurements of each sample were taken and averaged. The recovery of the analysis quality control standards and spike samples was $90-110 \%$, and the limit of detection for the procedure was $0.02 \mu \mathrm{g} / \mathrm{dL}$.

\section{Maternal bone lead}

To assess cumulative $\mathrm{Pb}$ exposure in mothers we used tibia bone $\mathrm{Pb}$ levels (half-life of approximately 10 years [19]) during the 1 month postpartum visit. Previous research demonstrates that pre-pregnancy bone lead levels are highly correlated with 1 month postpartum levels $[20,21]$. Maternal bone $\mathrm{Pb}$ was measured in the midtibial shaft using a K-shell X-ray fluorescence instrument (KXRF) [22]. Each leg was measured for $30 \mathrm{~min}$, and the results from both legs were averaged, weighted by the inverse of the measurement variance. Negative bone $\mathrm{Pb}$ values were replaced by sampling from a uniform distribution over the range of 0 to the DL $(2 \mu \mathrm{g} / \mathrm{g})$, as previously described [21, 23, 24].

\section{Infant saliva collection}

The saliva collection protocol (illustrated with photographs) was explained to mothers during the 12 month study visit. The collection material, an information diary and a printed copy of the protocol was provided to all participants who attended their study visit. If a mother 
Table 1 Comparison of Non-Participants and Participants

\begin{tabular}{llll}
\hline & $\begin{array}{l}\text { Non-participants } \\
n=355\end{array}$ & $\begin{array}{l}12 \text { month-old infants } \\
n=255\end{array}$ & $n=150$ \\
\hline Mother & & & month-old infants \\
Age at delivery (years) & $26.7 \pm 5.5$ & $27.1 \pm 5.6$ & $27.5 \pm 5.1$ \\
Education (total years) & $11.8 \pm 2.8$ & $11.9 \pm 2.8$ & $11.6 \pm 2.8$ \\
Pre-pregnancy BMl $\left(\mathrm{kg} / \mathrm{m}^{2}\right)$ & $24.9 \pm 4.1$ & $25.8 \pm 4.4^{*}$ & $24.7 \pm 3.9$ \\
2nd trimester blood lead $(\mu \mathrm{g} / \mathrm{dL})^{\mathrm{a}}$ & $3.6 \pm 2.5$ & $3.5 \pm 2.5$ & $3.9 \pm 2.8$ \\
3rd trimester blood lead $(\mu \mathrm{g} / \mathrm{dL})^{\mathrm{b}}$ & $3.8 \pm 2.7$ & $3.7 \pm 2.9$ & $4.2 \pm 3.4$ \\
Tibia lead $(\mu \mathrm{g} / \mathrm{g})^{c}$ & $4.7 \pm 5.4$ & $5.6 \pm 5.8$ & $4.9 \pm 5.0$ \\
Infant & & & $129(51)$ \\
Sex (male) $n(\%)$ & $182(51)$ & $224(88)$ & $90(60)$ \\
Ever breastfed $n(\%)^{d}$ & $303(85)$ & $38.3 \pm 1.5$ & $139(93)$ \\
Gestational Age, weeks & $38.3 \pm 1.9$ & $3.1 \pm 0.4$ & $38.5 \pm 1.6$ \\
Birth Weight, $\mathrm{kg}$ & $3.0 \pm 0.5$ & $3.2 \pm 0.4^{*}$ \\
\hline
\end{tabular}

For all analyses the imputed values for missing blood and tibia lead were used

anon-participant $n=354,18-24$ month-old infants $n=149$

${ }^{b}$ non-participant $n=301,12$ month-old infants $n=229,18-24$ month-old infants $n=139$

cnon-participant $n=270,12$ month-old infants $n=199,18-24$ month-old infants $n=117$

d non-participant $n=329,12$ month-old infants $n=, 18-24$ month-old infants $n=145$

*Unless noted $p>0.05$ for all comparison tests of covariates between non-participants and each age group

did not participate in the saliva collection following this visit, we inquired about participation at both 18 and 24 months as well. A total of 411 children provided saliva for cortisol at only one of these three visit dates (54\% completion rate).

Mothers were asked to collect 4 saliva samples per day from their child at home (early morning: after the infant woke up and had a diaper change but before breakfast, mid-morning: between 11:00 am and 1:00 pm, mid-afternoon: between 3:00 and 5:00 pm and night: at least 30 min after dinner, before bed time) for 2 days ( 8 samples total), and instructed that: collection days did not have to be consecutive but no more than a week apart, to wait at least $30 \mathrm{~min}$ before collecting the sample if the infant had been fed (including breastmilk), to collect samples on "typical" days (not days that were foreseen to be particularly stressful or busy), not to collect the samples if the infant was ill, taking medicine or had an allergic reaction to any food or insect bite.

Saliva was collected using a cotton braid which the infant was allowed to bite and suck between 10 and $30 \mathrm{~s}$. Saliva was then extracted using a needleless syringe to previously labeled collecting tubes on which the exact collection time was registered by the mother.

In the diary provided mothers were asked to record the specifics of collection for each sample, namely: the date for each collection day and times for the collection of each sample as well as for infant wake up, breakfast, dinner and bedtime. In all 8 times were recorded regarding the samples. Information on the child's sleep (whether the child slept enough (as usual) and if any naps were taken during the day registering the time of day and duration), and health (illness, medication name and time administered) particular to the sample collection day were also recoded.

Samples were stored in the participant's refrigerator until they were collected by our staff and then frozen at $-70{ }^{\circ} \mathrm{C}$ until shipment to the laboratory of the Technical University of Dresden, Germany for cortisol analysis. Saliva samples were analyzed in duplicate using a chemiluminescence-assay with a sensitivity of $\sim 0.16 \mathrm{ng} / \mathrm{ml}$ (IBL; Hamburg, Germany, Clemens Kirschbaum). Control samples covering at least three levels of cortisol were run for each day. The intra- and inter-assay coefficients of variation were less than $8 \%$.

We examined all the participants cortisol curves carefully, looking for any aberrant pattern. If we detected any irregularities, we consulted the diary for information on feeding, sleep or illness and excluded the sample(s) from our analyses if considered it relevant. Samples were also excluded if cortisol levels were greater than $3 \mathrm{SD}$ above or below the mean, when fever was reported and those for a 35 months-old infant. Only sampling days with at least 3 samples and with the wake up time registered were considered for the analyses. Our final sample included 3,110 cortisol samples (96\% of total collected samples) from 405 infants.

\section{Covariates}

Demographic information was obtained through standardized questionnaires. Potential covariates were identified based on the existing literature on $\mathrm{Pb}$ and cortisol 
response [25] as well as prior studies of cortisol rhythms performed in non-environmental health aims [17, 26, 27]: child's sex, gestational age, birth weight and breast-feeding (ever/never), maternal age at delivery, education (total years of school), pre-pregnancy BMI (self-reported weight and use of $2 \mathrm{~T}$ height), and weekday vs. weekend sample collection. Covariates were left out of the analyses if they were not associated with the exposure or outcome $(p>0.1)$ in bivariate models or if the cortisol effect estimates were not changed by more than $10 \%$. All models were adjusted for child's sex and maternal age at delivery, education and pre-pregnancy BMI.

\section{Statistical analysis}

We used longitudinal functional mixed effects regression models with penalized splines as described by Sanchez and colleagues [28]. This modeling framework accounts for the non-linearity of cortisol rhythms over the course of a day and addresses the multilevel structure in the data, whereby repeated measurements are taken within a day and measurements from multiple days are recorded for each participant [28]. The resulting models reflect how the association between exposure and cortisol levels varies as a function of "time since awakening" linked to a given cortisol measurement. Models can be run that assume that this association is constant across the course of the day "constant effect model" (CE), which implies that exposure is associated with a shift (increase or decrease) in the overall mean cortisol curve while maintaining the curve's shape. Alternatively, the model can assume that the association between exposure and cortisol level depends on the time since awakening "timevarying-effect model" (TVE), which allows changes in the shape of the cortisol curve. For the TVE model, results are illustrated graphically to present how the curve changes shape as a function of exposure. More details can be found in the Additional file 1. Cortisol concentrations were skewed to the left therefore we log-transformed them to better satisfy the normality assumption of the models.

In line with our aims, our initial analysis considered age as primary predictor of cortisol rhythms and this was statistically significant $(p>0.001)$. We then plotted the unadjusted cortisol curves for 12, 18 and 24 month old infants separately. Those of the 18- and 24-month old infants were very similar in shape and concentrations and not statistically different. Average cortisol levels in the 12-month old group were $21 \%$ higher (95\% CI $(9,34))$ than those of the 18-24-month infants (Fig. 1). Next, we ran models with an interaction term $\mathrm{Pb} \mathrm{x}$ exact-age-at-collection (we calculated the child's age in days using the difference between the birth date and the date when the saliva was sampled) which was statistically significant. Therefore, we subsequently ran separate prediction models for infants at 12 months and grouped 18-24 month old infants.

We defined cut-points to assess nonlinear relationships and whether an exposure threshold for $\mathrm{Pb}$ may be driving the associations. For bone $\mathrm{Pb}$, we used tertiles and for $\mathrm{BPb}$, we carried out analyses based on the updated $\mathrm{CDC}$ reference level for $\mathrm{BPb}$ of $5 \mu \mathrm{g} / \mathrm{dL}$ and the former level of $10 \mu \mathrm{g} / \mathrm{dL}$, which is the current reference level in Mexico according to the health norm NOM-199-SSA1-2000. We defined 3 categories of $\mathrm{BPb}$ exposure ("Lower" (Reference): $<5 \mu \mathrm{g} / \mathrm{dL}$; "Moderate": $5 \leq \mathrm{Pb}<10 \mu \mathrm{g} / \mathrm{dL}$; and "Higher": $\mathrm{Pb} \geq 10 \mu \mathrm{g} / \mathrm{dL}$ ).

In order to determine a prenatal window of susceptibility for $\mathrm{Pb}$ we regressed cortisol on 2 different $\mathrm{BPb}$ indicators (2nd trimester $-2 \mathrm{~T} \mathrm{BPb}$ and 3rd trimester-3 $\mathrm{T}$ $\mathrm{BPb}$ ) and an indicator of cumulative $\mathrm{Pb}$ exposure (maternal tibia bone $\mathrm{Pb}$ levels).

To account for missing $\mathrm{Pb}$ indicator data (see Table 1 for number of samples for each indicator), we used multiple imputation. We generated 10 data sets, which we used to run each of the models. For each model, the results of 10 imputations were averaged to give the final effect estimates. Standard errors were calculated using the methods that combine the within and betweenimputation uncertainty. We ran all our models with and without imputation and the results for the imputed models were consistent with the direction and magnitude of the associations. For the statistical analyses we used SAS 9.3 (SAS Institute, Inc., Cary, North Carolina,

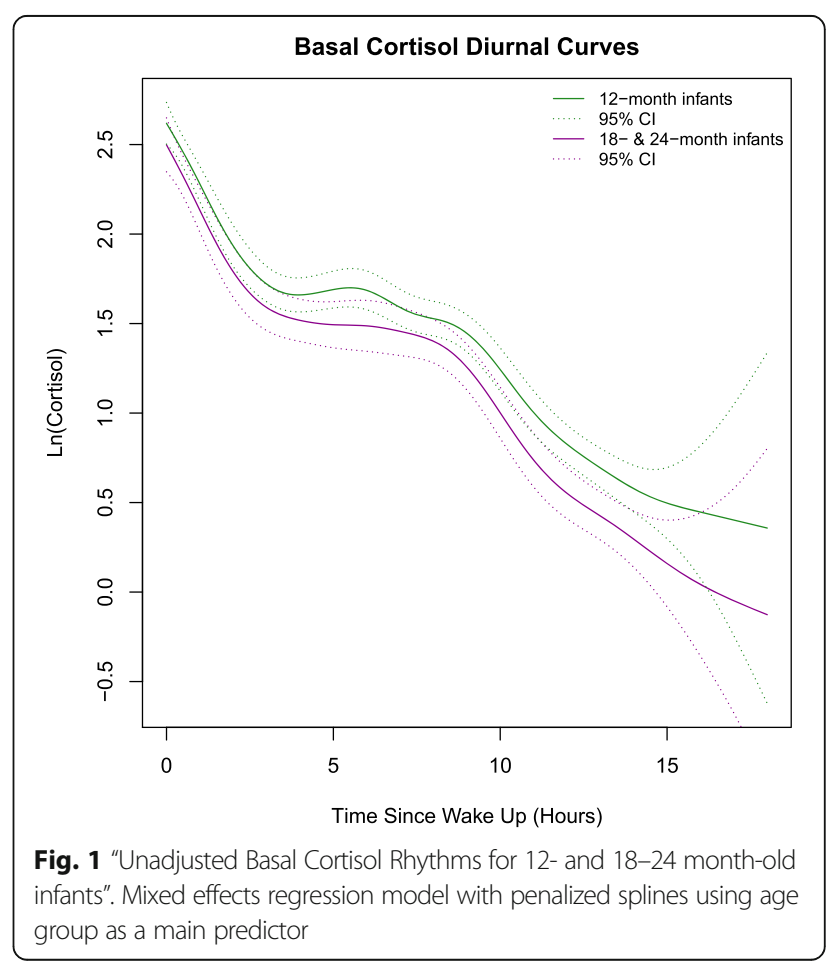


proc MI with the MCMC method for multiple imputation) and $\mathrm{R}$ version 3.0.2 with the gamm () function from the mgcv package for the functional mixed models [28].

\section{Results}

Age-dependent cortisol levels were confirmed by the results from the mixed effects regression model with penalized splines using age group (12- and 18-24-month-olds) as a main predictor. As reported above, younger children had higher basal cortisol levels throughout the day (Fig. 1). We found no statistically significant difference in saliva collection times between age groups. More information on cortisol concentrations by sample and for saliva collection times can be found in Table 1 of the Additional file 1 .

Results from Constant Effect Models (lead exposure not influencing the shape of the cortisol curve throughout the day) showed that among the 12-month old infants, mean cortisol levels for the moderate $(5 \leq \mathrm{Pb}$ $<10 \mu \mathrm{g} / \mathrm{dL})$ and higher $(\mathrm{Pb} \geq 10 \mu \mathrm{g} / \mathrm{dL}) \mathrm{Pb}$ exposure groups were lower compared to those in the referent category of $<5 \mu \mathrm{g} / \mathrm{dL}$ across $\mathrm{BPb}$ indicators (Table 2). The mean cortisol level for the higher- $2 \mathrm{~T}-\mathrm{BPb}$ was estimated to be $40 \%$ lower ( $95 \%$ CI $-57 \%,-16 \%$ ) than the mean cortisol level for the $2 \mathrm{~T}-\mathrm{BPb}$ referent group. Moderate- $3 \mathrm{~T}-\mathrm{BPb}$ was marginally associated with a 13 \% lower mean cortisol level (95\% CI $-27 \%$, 3 \%) compared to the mean cortisol level for the $3 \mathrm{~T}-\mathrm{BPb}$ referent group. This was contrary to our hypothesis that lead would be associated high higher cortisol levels. However, among the 18-24 month olds the associations between $\mathrm{Pb}$ exposure and cortisol were generally positive, although not statistically significant. Our results are clearer when presented graphically. As an example, Fig. 2 shows the results for the categorical $2 \mathrm{~T}-\mathrm{BPb}$ models stratified by age group. The adjusted natural log cortisol (lncortisol) curves illustrate the direction and magnitude of the associations with respect to the mean cortisol curve for each exposure category and for each age group.

Figure 3 shows the results for the Time Varying Effect (TVE)-models stratified by age, using the indicator for $2 \mathrm{~T}-\mathrm{BPb}$. These models allow for lead exposure to influence the cortisol level at any time point throughout the day, resulting in a change in the shape of the curve. The curves in Fig. 3 illustrate the estimated differences in mean (lncortisol) curves for the moderate- $\mathrm{Pb}$ and high$\mathrm{Pb}$ exposure groups, each relative to the low- $\mathrm{Pb}$ exposure group. For infants in both age groups, the mean curves associated with moderate- $2 \mathrm{~T}-\mathrm{BPb}$ exposure are the same shape as that for the corresponding low exposure group (no departure from the 0-change line). However, for both age groups the shape of the mean curve associated with high-2 $\mathrm{T}-\mathrm{BPb}$ exposure is modified relative to that for the low exposure group, with smaller differences occurring at the beginning of the day and larger differences occurring later in the day. This difference is significant $(p<0.05)$ among the 12-month-olds around midday (5-10 $\mathrm{h}$ post awakening). As in CE-models, these associations are inverse for the 12-month-olds and positive for the 18-24-month-olds. The results from models based on the other maternal $\mathrm{Pb}$ indicators were similar (data not shown).

Lastly, we confirmed that there might be a relevant window of susceptibility to lead and HPA axis development. The stronger and statistically significant association seen with $2 \mathrm{~T} \mathrm{BPb}$, specifically with higher $\mathrm{BPb}$ levels point at the susceptibility of this gestational stage for subsequent cortisol metabolism in infants.

\section{Discussion}

In this prospective birth-cohort study we found higher basal cortisol levels in 12 month-olds relative to 18-24

Table 2 Categorical analyses of the change on total diurnal infant (In) cortisol level stratified by infant age group

\begin{tabular}{|c|c|c|c|c|c|}
\hline & \multirow[t]{2}{*}{ Lower Lead $(<5 \mu \mathrm{g} / \mathrm{dL}$ ) } & \multicolumn{2}{|c|}{ Moderate Lead $(5 \leq P b<10 \mu \mathrm{g} / \mathrm{dL})$} & \multicolumn{2}{|c|}{ Higher Lead $(\geq 10 \mu \mathrm{g} / \mathrm{dL})$} \\
\hline & & $\beta$ & $\%$ change & $\beta$ & $\%$ change \\
\hline \multicolumn{6}{|l|}{ 12-Month Infants ${ }^{a}$} \\
\hline 2nd trimester & Ref & $-0.07(-0.24,0.10)$ & $-7(-22,10)$ & $-0.51(-0.85,-0.18)^{*}$ & $-40(-57,-16)^{*}$ \\
\hline 3rd trimester & Ref & $-0.14(-0.31,0.03)$ & $-13(-27,3)$ & $-0.02(-0.31,0.26)$ & $-2(-26,30)$ \\
\hline Tibia $^{c}$ & Ref & $0.02(-0.14,0.19)$ & $2(-13,20)$ & $-0.03(-0.21,0.14)$ & $-3(-19,15)$ \\
\hline \multicolumn{6}{|c|}{ 18- \& 24-Month Infants ${ }^{b}$} \\
\hline Second Trimester & Ref & $0.11(-0.08,0.30)$ & $12(-8,35)$ & $0.23(-0.19,0.65)$ & $26(-18,92)$ \\
\hline Third Trimester & Ref & $0.01(-0.17,0.20)$ & $1(-16,22)$ & $-0.05(-0.51,0.41)$ & $-5(-40,51)$ \\
\hline Tibia & Ref & $0.10(-0.13,0.32)$ & $10(-12,38)$ & $0.14(-0.08,0.35)$ & $14(-8,42)$ \\
\hline
\end{tabular}

Effect estimates: $\beta(95 \% \mathrm{Cl})$, \% change and (95\% Cl). All models are adjusted for child's gender and maternal age at delivery, pre-pregnancy BMI and total school years

$a_{n}=255$

${ }^{b} n=150$

ctertiles were used for tibia bone $(\mu \mathrm{g} / \mathrm{g})$

${ }^{*} p<0.05$, remained significant $(p<0.025)$ after Bonferroni correction for multiple comparisons 


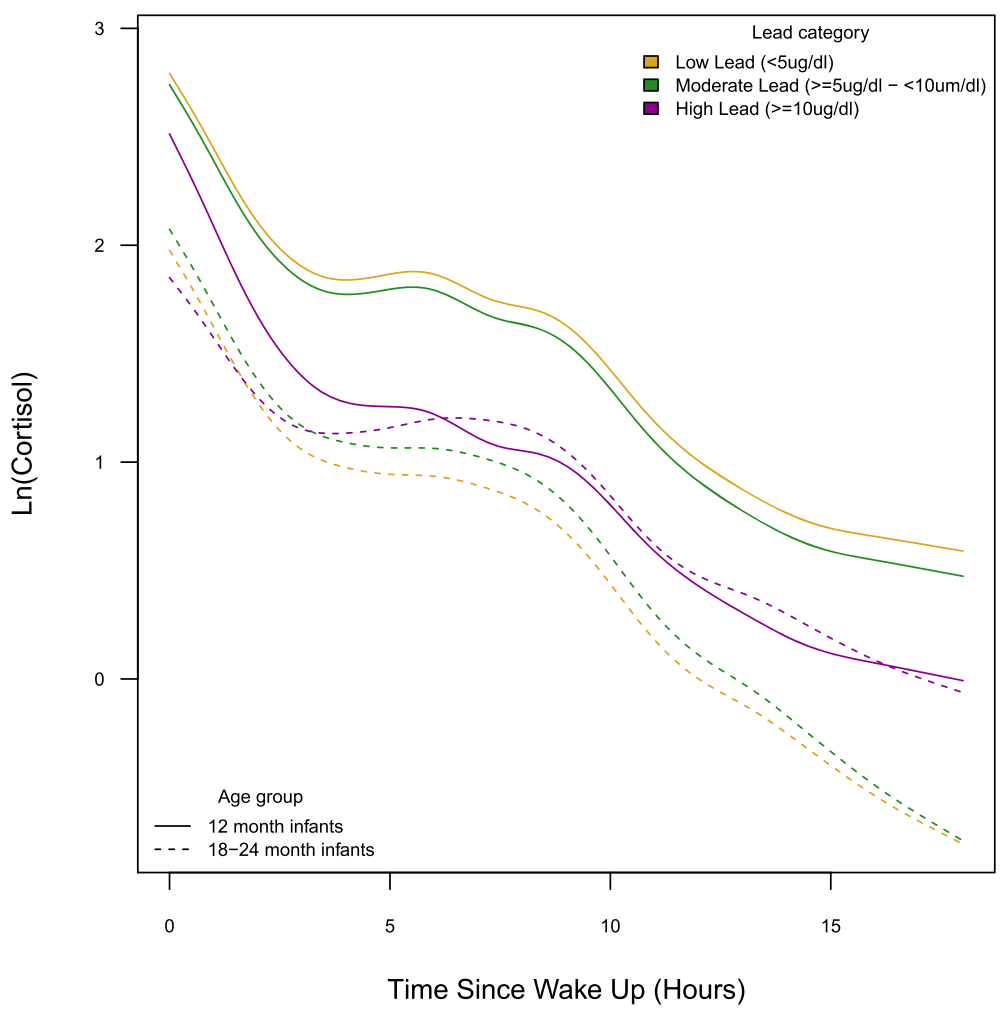

Fig. 2 "Effect of 2nd pregnancy trimester blood lead in categories on diurnal In(cortisol) curves stratified by age group (12 and 18-24-month-old)". All models were adjusted for child's gender and maternal age at delivery, pre-pregnancy BMl and total school years

month-olds which most likely represents the normal development of the HPA axis [17]. This information alone will be critical in planning future research on cortisol homeostasis as investigators should consider age at collection. Our results show that $\mathrm{Pb}$ exposure in pregnancy is associated with the infant cortisol curve differently based on the age at which the rhythm is measured. $\mathrm{BPb}$ was associated with a downshift in the cortisol curve at 12 months but with an upward shift in the cortisol curve at 18-24 months. Keeping in mind that the normal age trend from 12- to 18-24 months is a downshift, Pb exposure appears to produce a cortisol rhythm pattern in 12 month olds that is similar to the pattern seen in 18-24 month olds. This would be biologically-consistent with $\mathrm{Pb}$ driving a premature maturation of cortisol rhythm. Also, it appears that among 12-month-olds, higher prenatal $\mathrm{BPb}$ exposure modifies the shape of the cortisol rhythm. Among lower exposed children an age-dependent shift in the cortisol profile is seen and is likely a normal transition, but this shift is different if prenatal $\mathrm{BPb}$ is above $10 \mu \mathrm{g} / \mathrm{dL}$.

Cortisol is critical to neurogenesis and given Pb's wellknown effects on brain development, these results would be consistent with cortisol disruption being a mechanism mediating Pb's neurotoxicity, a hypothesis that has been studied in animal models. Corticosterone levels in rodent pups were altered by prenatal exposure to $\mathrm{Pb}$ in early pregnancy [29-32], but not when $\mathrm{Pb}$ exposure occurred in later gestational stages [33]; $\mathrm{Pb}$ accelerated age-related reductions in pre-stressor corticosterone levels in adult animals [29]; and there was a doseresponse relationship of prenatal $\mathrm{Pb}$ with cortisol levels [29]. Two other human studies have examined the adrenocortical response to stress in the context of $\mathrm{Pb}$ exposure, rather than basal cortisol rhythms and generally included older participants with presumably mature HPA axis function [13, 15]. In Gump et al. [14], investigators examined the association between prenatal and early childhood exposure to $\mathrm{Pb}$ and the cortisol response to an acute physical stressor in 9 year-old children. Both cord blood $\mathrm{Pb}$ and 2-year-old $\mathrm{BPb}$ measures were associated with an increased cortisol response to a cold immersion stress. Fortin et al. [13] examined the response to a psychological stressor among occupationally $\mathrm{Pb}$ exposed adult men. They found that higher $\mathrm{Pb}$ exposures were associated with lower basal cortisol levels [13]. Our results complement these findings, despite their methodologic differences, when taken as a whole, all indicate an association between $\mathrm{Pb}$ exposure and HPA axis disruption.

Our findings are in line with previous studies suggesting that $\mathrm{Pb}$ toxicity includes endocrine disruption. Given the role of cortisol in so many physiologic systems, the 


\section{Changes in the Shape of the Cortisol Curve}

Second Trimester Blood Lead

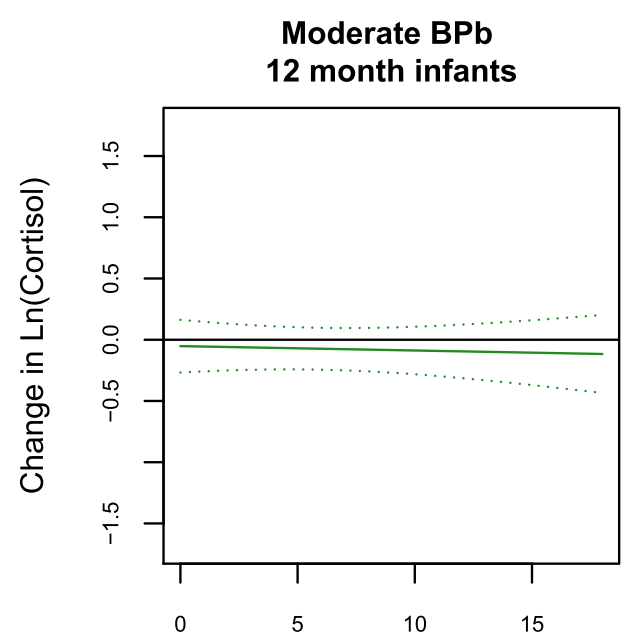

18-24 month infants

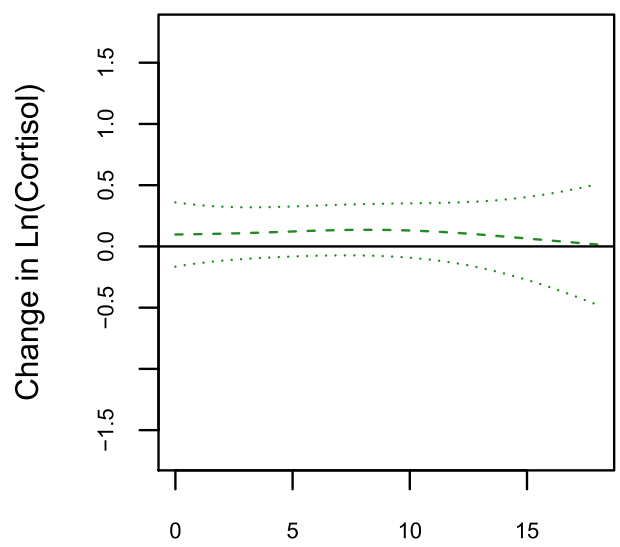

Time Since Wake Up (Hours)
High BPb

12 month infants

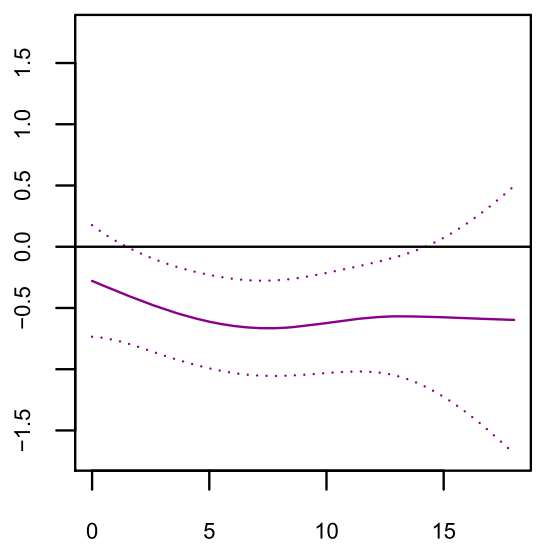

18-24 month infants

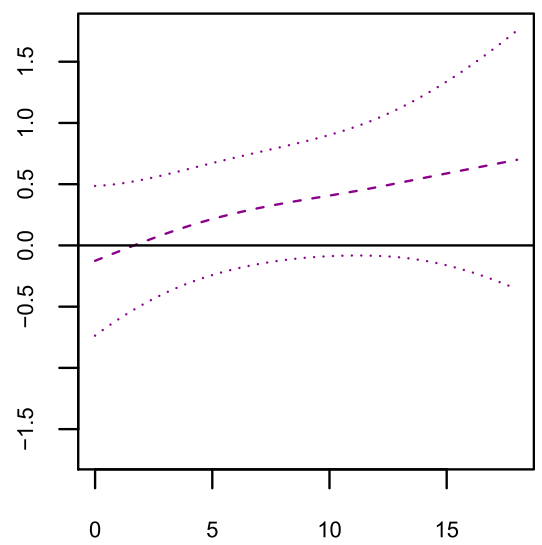

Time Since Wake Up (Hours)

Fig. 3 "Change in $\operatorname{In}($ cortisol) throughout the day associated with moderate $(5 \leq P b<10 \mu \mathrm{g} / \mathrm{dL}$ ) and high $(\geq 10 \mu \mathrm{g} / \mathrm{dL}$ ) 2nd trimester blood lead by age group". All models were adjusted for child's gender and maternal age at delivery, pre-pregnancy BMI and total school years

pleiotropic effect of $\mathrm{Pb}$ on other developmental processes, such as growth, cardiovascular and immunologic function, may also be related to disruptions of HPA axis function or maturation. Of note, the hippocampus, a well-known target of $\mathrm{Pb}$ toxicity and a central anatomic mediator of memory formation, has the highest concentration of glucocorticoid receptors in the central nervous system.

\section{Limitations}

We cannot rule out that infants might have experienced some stress during the sample collection however, any bias introduced by this however would be independent of prenatal $\mathrm{Pb}$ exposure which occurred $1-2$ years earlier and therefore could not confound our results. Our results are consistent with a maladaptive effect of prenatal $\mathrm{Pb}$ exposure on the HPA axis maturation but the implications of this must still be assessed. Our study considered only one cortisol rhythm measure, and longitudinal measures of salivary cortisol rhythms beyond 2 years of age may shed further light on this developmental trajectory.

\section{Conclusions}

To our knowledge, this is the first human study to look at the association of prenatal $\mathrm{Pb}$ exposure and infant basal diurnal cortisol rhythms as an index of HPA axis functioning. We found that age predicts lower basal cortisol secretion and that early prenatal $\mathrm{Pb}$ exposure is associated with dysregulated infant HPA axis function 
perhaps representing premature HPA axis maturation. More research is needed to confirm these findings. Long-term follow-up of these PROGRESS infants will allow additional insights into the possible health effects resulting from prenatal exposure to $\mathrm{Pb}$ and subsequent HPA axis disruption.

\section{Additional file}

Additional file 1: Details for statistical analysis method and Additional file 1: Table S1 "Salivary cortisol geometric means and sample characteristics by age group". (DOCX $18 \mathrm{~kb}$ )

\section{Abbreviations}

$2 \mathrm{~T}$ : second trimester of pregnancy; $3 \mathrm{~T}$ : third trimester of pregnancy; AUC: area under the curve; BMI: body mass index; BPb: blood lead; CAR: cortisol awakening response; CI-95 \%: confidence interval; CRYSIS: crisis in family systems; HPA: hypothalamic pituitary adrenal; IMSS: Instituto Mexicano del Seguro Social; Pb: lead; SD: standard deviation; SE: standard error.

\section{Competing interests}

The authors have no conflicts of interest to disclose and no financial relationships relevant to this article to disclose.

\section{Authors' contributions}

MTyO: Dr. Tamayo y Ortiz conceptualized and designed the study, carried out the analyses, drafted the initial and all consecutive versions of the manuscript, and approved the final manuscript as submitted. MMT-R, BAC and RW: Dr. Tellez-Rojo, Dr. Coull and Dr. RJ Wright helped in the conceptualization and design of the study. They helped carry out the analyses, reviewed and revised the manuscript, and approved the final manuscript as submitted. ROW: Dr. Wright is the P.I. of the PROGRESS birth cohort, helped in the conceptualization and design of the study, critically reviewed the manuscript and approved the final manuscript as submitted. All authors read and approved the final manuscript.

\section{Acknowledgements}

This work was supported by NIEHS grants R01 ES013744, R01 ES014930, P42 ES016454, P30 ES000002, P30 ES023515 and partially funded by the National Institute of Public Health/Ministry of Health of Mexico. We thank the Centro Médico $A B C$ and the National Institute of Perinatology, México for their support with this research.

\begin{abstract}
Author details
${ }^{1}$ National Council of Science and Technology, Center for Nutrition Research and Health, National Institute of Public Health, 7a. Cerrada de Fray Pedro de Gante \# 50, Col. Sección XVI Tlalpan, C.P. 14080 México D.F., Mexico. ${ }^{2}$ Center for Research in Nutrition and Health, National Institute of Public Health, Universidad No. 655, Col. Santa María Ahuacatitlán, Cerrada los Pinos y Caminera, CP. 62100 Cuernavaca, Morelos, Mexico. ${ }^{3}$ Departments of Pediatrics and Preventive Medicine, Pediatrics Kravis Children's Hospital, New York, USA. ${ }^{4}$ The Mindich Child Health \& Development Institute, Icahn School of Medicine at Mount Sinai, Annenberg Building Floor 14th Floor Room 8, 1468 Madison Avenue, New York City, NY 10029, USA. ${ }^{5}$ Department of Biostatistics, Harvard T.H. Chan School of Public Health, 655 Huntington Avenue, Building II, Room 413, Boston, MA 02115, USA. ${ }^{6}$ Department of Preventive Medicine, Icahn School of Medicine at Mount Sinai, 17 East 102 Street Floor 3 West Room D3-110, New York City, NY 10029, USA
\end{abstract}

Received: 10 September 2015 Accepted: 22 February 2016 Published online: 29 February 2016

\section{References}

1. Zoeller RT, Brown TR, Doan LL, Gore AC, Skakkebaek NE, Soto AM, et al. Endocrine-disrupting chemicals and public health protection: a statement of principles from The Endocrine Society. Endocrinology. 2012;153(9):4097110. doi:10.1210/en.2012-1422.
2. Vandenberg LN, Colborn T, Hayes TB, Heindel JJ, Jacobs Jr DR, Lee DH, et al. Hormones and endocrine-disrupting chemicals: low-dose effects and nonmonotonic dose responses. Endocr Rev. 2012;33(3):378-455. doi:10. 1210/er.2011-1050

3. Barouki R, Gluckman PD, Grandjean P, Hanson M, Heindel JJ. Developmental origins of non-communicable disease: implications for research and public health. Environ Health. 2012;11:42. doi:10.1186/1476-069X-11-42.

4. Smith SM, Vale WW. The role of the hypothalamic-pituitary-adrenal axis in neuroendocrine responses to stress. Dialogues Clin Neurosci. 2006;8(4):383-95

5. Afeiche M, Peterson KE, Sanchez BN, Cantonwine D, Lamadrid-Figueroa $H$, Schnaas $L$, et al. Prenatal lead exposure and weight of 0 - to 5-year-old children in Mexico city. Environ Health Perspect. 2011;119(10):1436-41. doi: 10.1289/ehp.1003184.

6. Cantonwine D, Hu H, Sanchez BN, Lamadrid-Figueroa H, Smith D, Ettinger AS, et al. Critical windows of fetal lead exposure: adverse impacts on length of gestation and risk of premature delivery. J Occup Environ Med. 2010; 52(11):1106-11. doi:10.1097/JOM.0b013e3181f86fee.

7. Ellis BJ, Boyce WT, Belsky J, Bakermans-Kranenburg MJ, van ljzendoorn MH. Differential susceptibility to the environment: an evolutionaryneurodevelopmental theory. Dev Psychopathol. 2011:23(1):7-28. doi:10. 1017/S0954579410000611.

8. Gore AC, Martien KM, Gagnidze K, Pfaff D. Implications of prenatal steroid perturbations for neurodevelopment, behavior, and autism. Endocr Rev. 2014;35(6):961-91. doi:10.1210/er.2013-1122

9. Bellinger DC. Prenatal exposures to environmental chemicals and children's neurodevelopment: An update. Saf Health Work. 2013;4(1):1-11. doi:10. 5491/SHAW.2013.4.1.1.

10. Granger DA, Blair C, Willoughby M, Kivlighan KT, Hibel LC, Fortunato CK, et al. Individual differences in salivary cortisol and alpha-amylase in mothers and their infants: relation to tobacco smoke exposure. Dev Psychobiol. 2007:49(7):692-701. doi:10.1002/dev.20247.

11. McDonald SD, Walker M, Perkins SL, Beyene J, Murphy K, Gibb W, et al. The effect of tobacco exposure on the fetal hypothalamic-pituitary-adrenal axis. BJOG. 2006;113(11):1289-95. doi:10.1111/j.1471-0528.2006.01089.x.

12. Kirlic N, Newman E, Lagasse LL, Derauf C, Shah R, Smith LM, et al. Cortisol reactivity in two-year-old children prenatally exposed to methamphetamine. J Stud Alcohol Drugs. 2013;74(3):447-51.

13. Fortin MC, Cory-Slechta DA, Ohman-Strickland P, Nwankwo C, Yanger TS, Todd AC, et al. Increased lead biomarker levels are associated with changes in hormonal response to stress in occupationally exposed male participants. Environ Health Perspect. 2012;120(2):278-83. doi:10.1289/ehp.1103873.

14. Gump BB, Reihman J, Stewart P, Lonky E, Granger DA, Matthews KA. Blood lead $(\mathrm{Pb})$ levels: further evidence for an environmental mechanism explaining the association between socioeconomic status and psychophysiological dysregulation in children. Health Psychol. 2009;28(5): 614-20. doi:10.1037/a0015611.

15. Gump BB, Stewart P, Reihman J. Low-level prenatal and postnatal blood lead exposure and adrenocortical responses to acute stress in children. Environ Health Perspect. 2008:116(2):249.

16. Caudle M. This can't be stressed enough: The contribution of select environmental toxicants to disruption of the stress circuitry and response. Physiol Behav. 2015. doi:10.1016/j.physbeh.2015.09.021.

17. Watamura SE, Donzella B, Kertes DA, Gunnar MR. Developmental changes in baseline cortisol activity in early childhood: relations with napping and effortful control. Dev Psychobiol. 2004;45(3):125-33. doi:10.1002/dev.20026.

18. Braun JM, Wright RJ, Just AC, Power MC, Tamayo YOM, Schnaas L, et al. Relationships between lead biomarkers and diurnal salivary cortisol indices in pregnant women from Mexico City: a crosssectional study. Environ Health. 2014;13(1):50. doi:10.1186/1476-069X-13-50.

19. Hu H, Rabinowitz M, Smith D. Bone lead as a biological marker in epidemiologic studies of chronic toxicity: conceptual paradigms. Environ Health Perspect. 1998;106(1):1-8.

20. Chuang HY, Schwartz J, Gonzales-Cossio T, Lugo MC, Palazuelos E, Aro A, et al. Interrelations of lead levels in bone, venous blood, and umbilical cord blood with exogenous lead exposure through maternal plasma lead in peripartum women. Environ Health Perspect. 2001;109(5):527-32.

21. Tellez-Rojo MM, Hernandez-Avila M, Lamadrid-Figueroa $H_{1}$ Smith D, Hernandez-Cadena L, Mercado A, et al. Impact of bone lead and bone 
resorption on plasma and whole blood lead levels during pregnancy. Am J Epidemiol. 2004;160(7):668-78. doi:10.1093/aje/kwh271.

22. $\mathrm{Hu} H$, Milder FL, Burger DE. The use of $\mathrm{KX}$-ray fluorescence for measuring lead burden in epidemiological studies: high and low lead burdens and measurement uncertainty. Environ Health Perspect. 1991;94:107-10.

23. Ganser GH, Hewett P. An accurate substitution method for analyzing censored data. J Occup Environ Hyg. 2010;7(4):233-44. doi:10.1080/ 15459621003609713.

24. Succop PA, Clark S, Chen M, Galke W. Imputation of data values that are less than a detection limit. J Occup Environ Hyg. 2004;1 (7):436-41. doi:10. 1080/15459620490462797.

25. Gump BB, Stewart P, Reihman J, Lonky E, Darvill T, Parsons PJ, et al. Lowlevel prenatal and postnatal blood lead exposure and adrenocortical responses to acute stress in children. Environ Health Perspect. 2008;116(2): 249-55. doi:10.1289/ehp.10391.

26. de Weerth C, Zijl RH, Buitelaar JK. Development of cortisol circadian rhythm in infancy. Early Hum Dev. 2003;73(1-2):39-52

27. Saridjan NS, Huizink AC, Koetsier JA, Jaddoe WW, Mackenbach JP, Hofman A, et al. Do social disadvantage and early family adversity affect the diurnal cortisol rhythm in infants? The Generation R Study. Horm Behav. 2010;57(2): 247-54. doi:10.1016/j.yhbeh.2009.12.001.

28. Sanchez BN, Wu M, Raghunathan TE, Diez-Roux AV. Modeling the salivary cortisol profile in population research: the multi-ethnic study of atherosclerosis. Am J Epidemiol. 2012;176(10):918-28. doi:10.1093/aje/kws182.

29. Cory-Slechta DA, Virgolini MB, Rossi-George A, Thiruchelvam M, Lisek R, Weston D. Lifetime consequences of combined maternal lead and stress. Basic Clin Pharmacol Toxicol. 2008;102(2):218-27. doi:10.1111/j.1742-7843. 2007.00189.x.

30. Cory-Slechta DA, Virgolini MB, Thiruchelvam M, Weston DD, Bauter MR. Maternal stress modulates the effects of developmental lead exposure. Environ Health Perspect. 2004;112(6):717-30.

31. White LD, Cory-Slechta DA, Gilbert ME, Tiffany-Castiglioni E, Zawia NH, Virgolini M, et al. New and evolving concepts in the neurotoxicology of lead. Toxicol Appl Pharmacol. 2007;225(1):1-27. doi:10.1016/j.taap.2007.08.001.

32. Rossi-George A, Virgolini MB, Weston D, Cory-Slechta DA. Alterations in glucocorticoid negative feedback following maternal $\mathrm{Pb}$, prenatal stress and the combination: a potential biological unifying mechanism for their corresponding disease profiles. Toxicol Appl Pharmacol. 2009;234(1):117-27. doi:10.1016/j.taap.2008.10.003

33. Graham DL, Grace CE, Braun AA, Schaefer TL, Skelton MR, Tang PH, et al. Effects of developmental stress and lead (Pb) on corticosterone after chronic and acute stress, brain monoamines, and blood $\mathrm{Pb}$ levels in rats. Int J Dev Neurosci. 2011;29(1):45-55. doi:10.1016/j.jjdevneu.2010.09.008.

\section{Submit your next manuscript to BioMed Central and we will help you at every step:}

- We accept pre-submission inquiries

- Our selector tool helps you to find the most relevant journal

- We provide round the clock customer support

- Convenient online submission

- Thorough peer review

- Inclusion in PubMed and all major indexing services

- Maximum visibility for your research

Submit your manuscript at www.biomedcentral.com/submit

C Biomed Central 